\title{
La contabilidad financiera como motor de la redefinición de la concepción de
} empresa*

\author{
Financial Accounting as a Driving Force to Redefine the Conception of Business \\ A contabilidade financeira como motor da redefinição da concepção de empresa
}

Steven Pardo-López a

DOI: https://doi.org/10.11144/Javeriana.cc18-46.cfmr

Institución Universitaria Politécnico Grancolombiano,

Colombia

stpardoll@poligran.edu.co

ORCID: http://orcid.org/0000-0003-4995-1459

Ángela Rocio Peña Cortés

Institución Universitaria Politécnico Grancolombiano,

Colombia

Fecha de recepción: 07 Agosto 2015

Fecha de aprobación: 07 Febrero 2017

Fecha de publicación: 15 Diciembre 2017

\section{Resumen:}

El predominio de la financiarización económica ha otorgado prevalencia a un concepto de empresa según el cual el accionista, la gestión financiera y la generación de valor emergen como elementos determinantes de las relaciones entabladas entre los agentes organizacionales. La reproducción de esta concepción en economías que carecen de estructuras financieras robustas constituye el centro de estudio del presente artículo. En este sentido, el trabajo explora el declive del sector industrial y el ascenso del sector financiero en Colombia. Asimismo, se identifica el surgimiento de tensiones entre la realidad organizacional y el ambiente institucional, por cuenta del ascenso de la concepción financiera de la empresa, en detrimento de la concepción orgánica de esta. El estudio concluye que estas tensiones son superadas con la ayuda de un isomorfismo institucional en el que las modernizaciones contables interpretan un papel central, puesto que insertan a la realidad organizacional un conjunto de técnicas y racionalidades afines a actividades especulativas.

Códigos JEL: M14, M41

Palabras clave: Empresa financiera, empresa orgánica, riesgo, control, isomorfismo.

\section{Abstract:}

The prevalent practice of the business financing has prioritized a business conception whereby the shareholder, financial management and value creation emerge as elements determining the relationship between the organizational agents. The reproduction of such conception in economies lacking strong financial structures then becomes the focus of this study. In this vein, this work explores the decline of the industrial sector and the rise of the financial sector in Colombia. Additionally, this work identifies the emerging tensions between the organizational reality and the institutional environment due to the rise of the business financial conception at the expense of the business organic conception. The study concludes that theses tensions can be overcome by using an institutional isomorphism in which the international accounting upgrades will play a central role. These upgrades will bring into the organizational reality a set of techniques and rationalities related to speculative activities.

Keywords: financial business, organic business, risk, control, isomorphism.

\section{Resumo:}

A predominância da financierização econômica tem dado prevalência para um conceito de empresa segundo o qual o acionista, a gestão financeira e a geração de valor emergem como elementos determinantes dos relacionamentos estabelecidos entre os agentes organizacionais. A reprodução desta concepção em economias que carecem de estruturas financeiras robustas constitui o centro de estudo do presente artigo. Nesse sentido, o trabalho explora o declínio do setor industrial e a ascensão do setor financeiro na Colômbia. Mesmo assim, identifica-se o surgimento de tensões entre a realidade organizacional e o ambiente institucional, devido a ascensão da concepção financeira da empresa, em detrimento da concepção orgânica dela. $\mathrm{O}$ estudo conclui que tais tensões são superadas com ajuda de um isomorfismo institucional em que as modernizações contáveis interpretam um papel central, pois inserem à realidade organizacional um conjunto de técnicas e racionalidades afins com atividades especulativas.

Notas de autor

\footnotetext{
${ }^{\text {a }}$ Autor de correspondencia. Correo electrónico: stpardoll@poligran.edu.co
} 
Palavras-chave: Empresa financeira, empresa orgânica, risco, controle, isomorfismo.

\section{Introducción}

La importancia de la actividad industrial en la estructuración del orden económico, de los siglos XIX y XX, permitió a la empresa productiva alcanzar un papel preponderante sobre otras unidades económicas como las agrícolas o comerciales. Esta situación estimuló la necesidad de comprender y analizar las interacciones dadas al interior de la empresa industrial como vía para optimizar el resultado económico y beneficiar a las clases capitalistas y obreras. En este punto, se impuso una visión generalizada según la cual la cooperación entre directivos y trabajadores constituía un elemento central para garantizar la eficiencia de las actividades productivas y el éxito del resultado empresarial.

No obstante, a partir la década de 1970, la preponderancia de la actividad industrial como motor de desarrollo económico y social empezó a ser eclipsada por el ascenso de la actividad financiera con fines especulativos. Lo anterior originó una redefinición del modelo de empresa contemporáneo, pasando de una visión orgánica hacia una visión financiera (Boyer, 2007); en donde los resultados organizacionales de cualquier actividad económica no solo se alcanzan por la combinación y la coordinación de sus factores productivos, sino también mediante la gestión de activos financieros que generan rendimientos en el corto plazo por medio de la especulación.

Tal redefinición, iniciada en los países anglosajones, se reprodujo con éxito en diversas regiones del mundo, en parte debido al apoyo político y económico encontrado en organismos supranacionales y al conjunto de reglas formales e informales que se consolidaron en torno de la lógica de la financiarización ${ }^{1}$ y del modelo económico que la origina.

En este escenario, una de las áreas del conocimiento que han tenido participación activa en la reconfiguración técnica y conceptual de las actividades organizacionales, en virtud de una visión financiera, ha sido la contabilidad; la cual, ha experimentado en las últimas décadas cambios en sus dimensiones disciplinar, práctica y regulatoria con efectos significativos en las dinámicas y concepciones empresariales.

En consecuencia, es nuestro interés resaltar cómo la modernización contable constituye una oportunidad para incentivar la redefinición de empresa. Lo anterior, a partir de la comprensión de la contabilidad como vía de comunicación bi-direccional entre la organización y su entorno, en donde: (a) permite a la organización capturar, interiorizar e instrumentalizar los supuestos que dominan el contexto en el que se desenvuelve y (b) incentiva una modelación del entorno organizacional, a partir de criterios individuales que adquieren un carácter objetivo ante la sociedad a partir del respaldo provisto por la contabilidad y su socialización a través de diversos canales de comunicación.

$\mathrm{Al}$ respecto, el contexto colombiano provee un escenario adecuado para ilustrar las tensiones institucionales que incentivan la redefinición financiera de la empresa, y el papel dinamizador que ocupa la contabilidad financiera. En ese sentido, se destacan reformas recientes que han modificado la estructura económica del país y que han permitido, entre otras cosas, el ascenso de la élite financiera doméstica; la reducción de las barreras del comercio internacional; el crecimiento y la integración de los mercados financieros, y la adopción del modelo de gobierno de empresa anglosajón. En el marco de ese conjunto de reformas, la adopción de Normas Internacionales de Información Financiera - en adelante NIIF - se perfila como un aspecto clave para el establecimiento de la definición financiera de la empresa como una perspectiva dominante a nivel nacional.

Con el objetivo de desarrollar la exploración realizada hasta este punto, el documento ha sido estructurado en cinco secciones. La primera está constituida por la presente introducción. Por su parte, la segunda sección describe el declive del sector industrial y el auge del sector financiero en el contexto colombiano; asimismo, caracteriza las concepciones orgánica y financiera de la empresa. En la tercera sección se evidencian las tensiones que se tejen entre la realidad organizacional y el ambiente institucional colombiano, por cuenta de 
la concepción dominante de empresa; además, se introduce el isomorfismo institucional como la vía a partir de la cual se normalizan la concepción financiera de la empresa y la gestión de riesgos. En tanto, la cuarta sección evidencia algunas modernizaciones contables que se perfilan como elementos activos que refuerzan el isomorfismo en el que se institucionalizan prácticas organizacionales afines al contexto financiarizado. Por último, la quinta sección presenta las conclusiones del estudio.

\section{La concepción orgánica y la concepción financiera de la firma}

La perspectiva economista de desarrollo, que mantiene su supremacía desde la primera Revolución Industrial hasta nuestros días, plantea desarrollo como crecimiento económico; a partir de la teoría económica neoliberal y el establecimiento del sistema capitalista, hay una concepción del crecimiento económico como la acumulación de capital que permite la actividad empresarial y de la distribución de riqueza como el flujo de bienes que se da a través de las relaciones de mercado.

Bajo dicha perspectiva, la empresa es la unidad básica de desarrollo capitalista que permite a los individuos gestionar su crecimiento económico y contribuir al bienestar social. En medio de su rol protagónico, esta ha sido sujeto de importantes transformaciones en cuanto a su concepción por parte de los agentes que la componen y se relacionan con ella, en especial, después de la segunda mitad del siglo XX.

$\mathrm{Al}$ respecto, autores como Boyer (2007) y Aglietta y Rebérioux (2009) han caracterizado dos concepciones generales de empresa, que son al tiempo, dos visiones para articular y gestionar los factores productivos que la involucran. La primera es la concepción orgánica de la firma, la cual se asocia con un modelo de empresa industrial hegemónico entre 1840 y 1970 . La segunda es la concepción financiera de la firma, la cual se vincula con un modelo financiarizado, y en general con una hegemonía creciente desde la década de 1970. Con base en la evolución del modelo empresarial en Colombia, a continuación se ilustrarán las características más importantes de las dos concepciones de empresa reseñadas.

\section{El modelo industrial como impulsor del desarrollo económico}

En el periodo comprendido entre 1840 y 1970, el ideal de desarrollo económico de las naciones occidentales se fundamentó en la tecnificación del sector industrial y en la plena libertad para la acumulación de capital a partir de la propiedad privada. En las primeras décadas de esta etapa de industrialización, se forjó la renovación del orden político-económico internacional; en donde se estableció para 1930, una estructura conformada por los países totalmente industrializados y los países en industrialización tardía. Posteriormente, la Segunda Guerra Mundial propició un ambiente de nacionalización parcial de las economías para enfrentar los problemas de comercio internacional — como la escasez de bienes importados, o la sobreproducción de bienes exportables - surgidos por efectos del conflicto.

En el periodo de posguerra, para el caso de los países con industrialización tardía, resalta una profundización del nacionalismo económico como un intento de seguimiento a la estrategia de industrialización que aplicaron algunos países occidentales antes de 1930. Como principal efecto de dicha tendencia, se institucionaliza en Latinoamérica el modelo de industrialización por sustitución de importaciones (ISI). ${ }^{2}$ Según Misas (1998), el modelo se caracterizó principalmente por la ejecución de tres estrategias claves orientadas hacia el fortalecimiento de la industria nacional: (a) el desincentivo de la importación por medio del aumento de las tasas arancelarias; (b) la producción interna de bienes sustitutos, y (c) el control del flujo de capital extranjero por medio de una alta tasa cambiara. 


\section{Auge y declive del modelo industrial en Colombia}

En coherencia con el modelo ISI, en las décadas posteriores a la Segunda Guerra Mundial, Colombia buscó que la industria fungiera como un polo de desarrollo que permitiera expandir el crecimiento económico a lo largo de toda la cadena productiva. Al igual que en el marco regional, la industrialización de la empresa nacional se caracterizó por la producción de bienes con precios relativos altos, debido a una base tecnológica atrasada; situación que se corregía, parcialmente, por los efectos de las políticas cambiarias que devaluaban la moneda para desalentar la importación; efecto que, no obstante, llevaba a la economía a una etapa de recesión en la que las empresas debían ejercer restructuraciones de la planta de personal, facilitando las exportaciones por precios bajos (Frieden, 2006).

El modelo industrial desarrollado durante esta etapa, llevó a la industrialización del país a un nivel de ventaja comparativa mínima frente a otros países con mejores resultados como Brasil y México. En parte, tal situación se explica por el atraso tecnológico, la ausencia de mano de obra altamente capacitada ${ }^{3}$ y la incapacidad para aumentar la empleabilidad. No obstante, a pesar de las limitaciones que exhibió el modelo, el país logró articular los factores productivos para cumplir con los propósitos internos de demanda y oferta.

Los antecedentes del declive del modelo ISI inician en la década de 1960. López (2010) afirma que en aquella época la demanda de productos comenzó a ser limitada a nivel intranacional, por lo que el problema de industrialización en productos no transables y concentrados en un modelo agroexportador, reclamó un cambio en la política económica colombiana. Paralelamente, las políticas de libre comercio, impulsadas en los organismos supranacionales por parte de los países desarrollados, afectaron el proteccionismo en el que estaba sumida Latinoamérica. Así, la combinación de presiones internas y externas sobre el modelo llevó a que este fuera desmontado a partir de la década de 1970, fecha en la cual comenzó la etapa de preapertura de mercado en el país.

Dicha etapa de preapertura coincide con la caída del Sistema Bretton Woods, en la cual el Fondo Monetario Internacional (FMI) promulgó el sistema de libre flujo de capital financiero como requisito para el establecimiento de un sistema de comercio internacional perfecto. De ese modo, logró que los países flexibilizaran su política cambiaria, y enfrentaran el cambio del sistema dólar-oro. En busca de una respuesta a los mensajes provenientes de los organismos multilaterales, el país se embarcó en una política semiproteccionista que permitiera aumentar la competitividad de la industria desarrollada; que incrementara la participación del sector servicios, y que lograra un apalancamiento de ambos sectores en el sector financiero (López, 2010). Sin embargo, la baja capacidad de producción de bienes con alta concentración en capital generó una dependencia creciente hacia los mercados extranjeros para obtener bienes con alto valor agregado y atraer proveedores de tecnología y capital financiero.

La apertura total de la economía nacional, a comienzos de la década de 1990, fortaleció la dinámica de acuerdos multilaterales. En dicho marco, la liberalización de flujos de capital financiero y de bienes reales de la economía creó un escenario en el que los grandes grupos económicos que habían monopolizado el sector manufacturero reafirmaron su posición oligopólica. Lo mismo sucedió con las empresas con inversión extranjera directa, las cuales mantuvieron la práctica de fuga de capitales.

Los grupos económicos ganaron, pues la mayoría de activos [...] concentrados en sectores productores no transables y, con las medidas de desregularización y liberalización, pudieron apropiarse de grandes excedentes en la prestación de servicios, tales como energía, telecomunicaciones y finanzas. (Misas, 1998, p. 337)

En este sentido, el declive de la industria nacional se marca desde 1970 hasta la actualidad. La transformación del país hacia el desarrollo industrial fue un camino truncado tanto por las limitaciones internas para desarrollar una producción con mayor valor agregado, como por la inclinación internacional hacia un nuevo modelo de desarrollo economicista: primero de comercio internacional y ahora financiarizador, que alejaron al país de una mejor consolidación de su modelo industrial. Después de la década 
de 1970, el sector industrial no presentó avances significativos en materia de diversificación (López, 2010). En consecuencia, se caracteriza en la actualidad por su mano de obra no tecnificada, y por sostener a sus sectores manufactureros y agroexportadores, que se beneficiaron en los años 1950 con el modelo ISI, en una prolongada crisis.

\section{Concepción orgánica de la empresa}

Como precursora del desarrollo nacional, la empresa industrial fue el centro de las teorías de gestión de la organización, y determinó las relaciones de los agentes involucrados con ella, al igual que el uso y los fines de los recursos disponibles. Bajo el modelo de la empresa industrial, la acumulación de riqueza solo era posible mediante las relaciones de producción y consumo de los bienes reales. En consecuencia, esta era vista como un sistema de entrada de recursos o factores de producción, y de salida de bienes disponibles para un mercado perfecto; es decir había una concepción de la empresa como un organismo en el que fluyen los recursos y se trasforman en valor agregado. A continuación se describen las principales características de esta concepción de empresa:

1. El propietario y/o gerente como figura central de la organización. La eficiencia y la competitividad organizacional necesarias para generar valor productivo constante, así como para facilitarle la acumulación de riqueza al dueño de la firma, se alcanzan con la ejecución de estrategias especializadas en producción a gran escala, en búsqueda de expansión del mercado y un mejor relacionamiento con los agentes de la cadena productiva. El gerente, surge como aquel agente especializado que procura estrategias para el uso racional y eficiente de los recursos y la maximización de las utilidades.

En algunos escenarios, el papel del gerente es asumido por el mismo propietario, en virtud de su elevado conocimiento sobre la actividad operativa de la organización. Lo anterior sucede especialmente en países en los cuales la empresa familiar es prominente.

2. La generación de utilidades es el camino utilizado para la generación de riqueza y para la acumulación de capital. El valor de la empresa está asociado directamente con los rendimientos vía utilidades; la generación de ingresos que se logra a través de la combinación de los factores de producción brinda estabilidad financiera a la organización en el mediano plazo. Asimismo, la capacidad de realizar una buena inversión de los rendimientos, primordialmente en más factores de producción, genera estabilidad financiera a largo plazo, y sustenta la generación de riqueza. Con base en esto, es posible inferir que el valor empresarial está directamente relacionado con la cadena productiva, y que los indicadores de desempeño de las organizaciones se basan en una relación positiva de costo-beneficio. Además, los modelos contables asociados a esta medición del desempeño se basan en valores que permiten identificar la eficiencia en la transformación de los factores de producción y la estabilidad financiera de acuerdo con las utilidades alcanzadas en el ciclo económico. En consecuencia, solo dan cuenta del valor generado.

3. La gestión financiera apoya el desarrollo de la gestión productiva de la organización. El uso eficiente del capital industrial es el centro de los modelos de gestión, mientras el capital financiero funge como medio para garantizar la propiedad privada de los activos productivos. La toma de decisiones en la concepción orgánica de la empresa, establece el recurso financiero como un medio para la generación de utilidades, vía fortalecimiento de la capacidad productiva.

Los modelos contables basados en datos de entrada evidencian la relación que existe entre la gestión financiera y la gestión productiva, a la luz de la concepción orgánica de la empresa. Estos modelos anteponen la circulación económica real ante la circulación financiera, pues normalizan un conjunto de prácticas que principalmente describen la entrada de factores de producción, su 
transformación, su salida y su venta; así, relegan a una segunda instancia la descripción del capital financiero en función de su vínculo con todo el proceso productivo (Cañibano, 1988; Aglietta y Rebérioux, 2009). En este escenario, técnicas de valoración como el costo histórico permiten a la contabilidad atender las necesidades de control en organizaciones afines a una gestión de recursos en la que los movimientos financieros dependan de los movimientos del recurso real.

\section{Ascenso de la esfera financiera}

Los procesos de globalización y liberalización financiera han incidido en la modelación de varias características del contexto organizacional contemporáneo. En ese sentido, se destaca la forma como el predominio de las finanzas y, en particular, de los mercados financieros, ha redefinido los vínculos entre las organizaciones y el sistema financiero, con base en prácticas administrativas y organizacionales que facilitan la penetración de la lógica del capital financiero en compañías no financieras (Zhang y Andrew, 2014). Lo anterior se desarrolla en el marco de la Nueva Arquitectura Financiera Internacional (NAFI) y de múltiples procesos de estandarización internacional, los cuales han sido impulsados por los países desarrollados - especialmente los anglosajones-, que resaltan la concepción financiera de la firma por encima de la concepción orgánica de esta.

Cabe añadir que, en el marco del desarrollo económico contemporáneo, el empoderamiento de las finanzas responde a un modelo de acumulación capitalista denominado financiarización, caracterizado por "la dominación de la maximización del valor para los accionistas como el objetivo corporativo por encima de todo" (Guttman, 2009, p. 22), en donde la búsqueda de rendimientos de corto plazo se antepone a la búsqueda de rendimientos de largo plazo, y se rediseña la organización interna de las empresas en función de las demandas de la rentabilidad bursátil (Giraldo, 2007).

En el sentido descrito, los países latinoamericanos constituyen escenarios en los que se han presentado cambios estructurales en materia de política económica. Dichos cambios fortalecen la aparición de condiciones adecuadas para la redefinición del concepto dominante de empresa, en consonancia con los incentivos que plantea la creciente complejidad e integración de los mercados financieros.

Con el fin de ilustrar la relación existente entre la consolidación del sistema financiero y el fortalecimiento de la perspectiva financiera de la firma, en el siguiente apartado se buscará describir el contexto de las reformas al sistema financiero colombiano. Asimismo, se abordarán las características más sobresalientes de la concepción financiera de la empresa.

\section{La transformación del Sistema Financiero colombiano}

La importancia del sistema financiero en el devenir de las organizaciones es indiscutible. En ese sentido, el acceso a fuentes de financiación permite que las organizaciones ejecuten proyectos propios de su actividad generadora de renta; acciones que redundan en la obtención de los objetivos planteados por la administración. No obstante, las vías de cooperación entre ambos elementos (el sistema financiero y las empresas) son dinámicas. Además, varían con frecuencia en función del tiempo y del espacio.

En función del tiempo, en la medida en que la historia financiera del siglo XX evidencia una evolución caracterizada por la complejización y la integración de las estructuras financieras nacionales, especialmente, con la ayuda de las Tecnologías de la Información y la Comunicación (TICs), en donde la banca especializada perdió protagonismo, en virtud de la multibanca, la cual a su vez cedió terreno ante el ascenso de los inversionistas institucionales, que intervinieron de la mano de los mercados de capitales (Garcia y Gómez, 2009). 
En función del espacio, en la medida en que las áreas geográficas han supuesto prácticas de gestión empresarial particulares, que determinan las fuentes de financiación predilectas por las empresas de la región. Así, encontramos países como Alemania y Francia, en los cuales la banca privada ha jugado un papel preponderante para la financiación de las actividades empresariales; sin embargo, también existen modelos como el inglés o el norteamericano, en los que las organizaciones han privilegiado a los mercados de capitales como principales fuentes de liquidez (Aglietta y Rebérioux, 2009; Pardo, 2014) y escenarios como Latinoamérica, en el que el patrimonio ha representado históricamente la principal fuente de financiación empresarial.

En el caso de Colombia, aunque ya ha quedado claro que entre las décadas de 1970 y 1980 se efectuaron algunos cambios en materia de política económica a favor de la reforma neoliberal, la primera mitad de la década de 1990 fue el periodo que constituyó el punto de inflexión del nuevo direccionamiento económico del país. Para entonces, se realizaron reformas a la legislación laboral, los regímenes cambiarios, el sistema financiero, el sistema de salud y el sistema pensional, entre otros (López, 1995). ${ }^{4}$

Estos cambios en materia de política económica propiciaron un escenario coherente con el contexto internacional; un contexto en el que "el apoyo a las instituciones financieras y la integridad del sistema financiero se convirtió en la principal preocupación de la colectividad de los Estados" (Harvey, 2006, citado por Zhang y Andrew, 2014, p. 19). De esa manera, cambios como la eliminación de barreras para el ingreso de capital extranjero, la venta de entidades bancarias propiedad del Estado, ${ }^{5}$ y la concentración del ahorro de los trabajadores por parte de inversionistas institucionales, brindaron mayor volumen de recursos al sistema financiero. Dichos recursos permitieron a su vez el aumento de la oferta de instrumentos de financiación disponibles para las empresas y los hogares.

La expansión del sistema financiero colombiano, dada mediante la liberalización de mercados, ha continuado hasta la actualidad con acuerdos como la integración regional de las bolsas valores de Colombia, Perú y Chile - conocida como el Mercado Integrado Latinoamericano (MILA) —; la reducción de las barreras a la movilidad de capitales mediante los tratados de libre comercio (TLCs), y la flexibilización de las condiciones de inversión de los fondos de pensiones privados o de las condiciones tributarias que favorecen las rentas de capital.

En los contextos económicos y financieros que han caracterizado el devenir del país durante las dos décadas y media más recientes, estos cambios han propiciado el redireccionamiento de algunas prácticas organizacionales que resultan notorias. Lo anterior ha sido conseguido, por ejemplo, con (a) la adopción de prácticas de gobierno corporativo anglosajonas, tras la Resolución 275 de 2001 y la Ley 964 de 2005 (Banco Interamericano de Desarrollo y Bolsa de Valores de Colombia [BID y BVC], 2009; Gaitán, 2010); (b) la adopción de estándares internacionales en contabilidad y auditoría tras la Ley 1314 de 2009; (c) la creciente acogida voluntaria de los estándares consignados en el modelo de la Organización Internacional de Estandarización (ISO, por sus siglas en inglés), o (d) la divulgación de información social-ambiental, mediante el estándar de la Iniciativa de Reporte Global (GRI, por sus siglas en inglés).

Estas nuevas prácticas han fomentado la competencia entre las actividades especulativas y operativas al interior de la organización; transformaciones surgidas tras la introducción de modelos de comportamiento alineados con criterios e intereses inversionistas. Así, se observa un paulatino desplazamiento de la concepción de empresa, la cual se aboca hacia una definición financiera en la cual la aplicación de estrategias que permiten reducir los costos de transacción y producción - bajo un concepto de eficiencia financiera antes que productiva - incursionan como prácticas comunes en la gestión organizacional (Boyer, 2007; García y Gómez, 2009). En tanto, la empresa es considerada con mayor frecuencia un objeto que garantiza un retorno mínimo a la inversión del accionista y, en consecuencia, sus resultados pueden ser comparados y reemplazados por activos financieros que generan una mayor tasa de retorno (Lordon, 2007). 


\section{Definición financiera de la empresa}

La definición financiera de la empresa responde a un contexto financiarizado en el que los incentivos han modificado el sentido de las relaciones entabladas entre organizaciones y mercados financieros, con lo que se deja en un segundo plano el modelo orgánico de las organizaciones. En este escenario, el papel de los instrumentos financieros cambia; estos dejan de concebirse exclusivamente como medios que permiten la financiación de actividades operativas, en virtud de una perspectiva según la cual también se constituyen como eventuales fuentes de utilidades. A continuación, se describen las principales características de esta concepción de empresa.

1. El accionista se transforma en la figura central de la organización. La noción financiera de la empresa ubica al accionista como el stakeholder preponderante de la organización, por cuenta de su papel como proveedor de capital financiero. De esta forma, el accionista legitima su primacía, bajo el argumento de que la remuneración que obtiene es un residuo, el cual es el resultado de deducir las retribuciones a los demás agentes organizacionales (trabajadores, proveedores, Estado, etc.). No obstante, este argumento desconoce la capacidad de movilidad del accionista como inversor. Un rol bajo el cual es usual que la transferencia de acciones constituya en el corto plazo una vía para eludir el riesgo que supone la operación de la empresa.

Bajo la perspectiva financiera de la empresa, el accionista es el agente que corre los mayores riesgos.

${ }^{6}$ Como retribución, tiene derecho a controlar las actividades que se lleven a cabo al interior de la organización (Aglietta y Rebérioux, 2009).

2. La generación de valor accionarial se privilegia por encima de la generación de utilidades. El desplazamiento de la concepción orgánica a la concepción financiera de la empresa supone un tránsito de la utilidad a la generación de valor accionarial como principal indicador del desempeño organizacional e, incluso, como un indicador de ingresos para los agentes (Rayman, 2007; Lordon, 2007). De esa manera, las prácticas contables y financieras ${ }^{7}$ que favorecen la valoración de la firma como una suma de activos desestructurados prevalecen sobre las prácticas que valoran la firma desde una perspectiva holista.

En este escenario, la información que permite predecir las eventuales variaciones del valor accionarial se tipifica como relevante, y se convierte en un elemento decisivo para la toma de decisiones de los usuarios de la información financiera.

3. La gestión operativa de la empresa se ve eclipsada por la gestión financiera. Dado que la coordinación intrafirma pierde importancia en la definición financiera de la empresa, la gestión operativa resulta eclipsada por la gestión financiera (Crotty, 2003). Así, tanto la eficiencia del proceso productivo como la reducción de los costos operacionales, implementadas para garantizar la maximización de las utilidades, empiezan a ser objetivos de segundo nivel. En su lugar, la eficiencia de la gestión de los activos y pasivos que permiten la creación de valor accionarial y la mejora de indicadores como el valor económico agregado (EVA, por sus siglas en inglés) se convierten en el principal objetivo de la administración de la compañía (Rayman, 2007; Aglietta y Rebérioux, 2009). Dentro de las prácticas que despiertan especial atención de la gestión financiera, se destacan (a) la reducción del costo del capital por medio de la búsqueda de fuentes de financiación externas; (b) el aumento de la rentabilidad con base en ganancias no realizadas, o (c) la gestión financiera del riesgo como vía para incrementar el valor presente de los activos.

Los modelos contables basados en datos de salida evidencian la relación existente entre la gestión financiera y la gestión organizacional a la luz de la concepción financiera de la empresa. Estos modelos anteponen la circulación financiera a la circulación económica real, al normalizar prácticas que se enfocan en la descripción de posibles flujos de caja futuros; además, relegan a un 
segundo plano las transacciones reales que han definido el estado actual de los recursos (Aglietta y Rebérioux, 2009). En este escenario, técnicas de valoración como el valor razonable le permiten a la contabilidad atender las necesidades de control de organizaciones afines a una gestión de recursos en la que los movimientos financieros y especulativos definen los movimientos del recurso real.

De acuerdo con el estudio de la evolución del mercado económico nacional y de las políticas económicas que han propiciado los hitos de cambio, el contraste que se nota entre el declive del sector industrial y el ascenso del sector financiero permite pensar que a nivel macroeconómico existe un escenario que propicia el desplazamiento de la concepción dominante de la empresa (ver Tabla 1). Asimismo, pone en evidencia el escalamiento de las dinámicas de la financiarización; y además demuestra que a nivel local no se perciben resistencias significativas a las dinámicas internacionales de mercado y gestión de las organizaciones. No obstante, también conduce a la necesidad de indagar sobre las formas como se institucionalizan los preceptos de la concepción financiera de la empresa, así como los procesos organizacionales que los soportan a nivel intrafirma.

TABLA 1

Concepción orgánica vs. concepción financiera de la empresa

\begin{tabular}{|c|c|c|}
\hline \multicolumn{3}{|c|}{ Concepción orgánica vs. concepción financiera de la empresa } \\
\hline Caracteristicas del modelo de gestión & Concepción orgánica & Concepción financiera \\
\hline $\begin{array}{l}\text { Agente principal para la toma de } \\
\text { decisiones }\end{array}$ & $\begin{array}{l}\text { Gerente: en los casos de las grandes empresas } \\
\text { Propietario: en los casos las empresas familiares }\end{array}$ & $\begin{array}{l}\text { Accionista } \\
\text { Es notorio el problema en la concepción de la dualidad accionista } \\
\text { / inversor. }\end{array}$ \\
\hline Interés en el mantenimiento del capital & Productivo & Financiero \\
\hline Generación de excedentes económicos & Utilidades & Rentabilidades \\
\hline $\begin{array}{l}\text { Tiempo esperado del excedente } \\
\text { económico }\end{array}$ & Mediano-largo plazo & Corto plazo \\
\hline $\begin{array}{l}\text { Gestión del recurso principal para la } \\
\text { obtención del excedente económico }\end{array}$ & $\begin{array}{l}\text { Bien real } \\
\text { Reducción de costos por medio de la correcta } \\
\text { estructuración de los activos productivos. }\end{array}$ & $\begin{array}{l}\text { Instrumentos financieros } \\
\text { Reducción de costos por medio de: } \\
\text { (1) La reducción del costo del capital mediante la búsqueda de } \\
\text { fuentes de financiación externas. } \\
\text { (2) El aumento de la rentabilidad con base en ganancias no } \\
\text { realizadas. } \\
\text { (3) La gestión del riesgo como via para incrementar el valor } \\
\text { presente de los activos. }\end{array}$ \\
\hline $\begin{array}{l}\text { Mercado objetivo para la obtención del } \\
\text { excedente económico }\end{array}$ & Mercado económico & Mercado financiero \\
\hline $\begin{array}{l}\text { Modelo contable para la medición / } \\
\text { valoración del excedente económico }\end{array}$ & $\begin{array}{l}\text { Centrado en las operaciones de la economía real. } \\
\text { Uso de métodos de valoración de entrada (prevalencia } \\
\text { del costo histórico). }\end{array}$ & $\begin{array}{l}\text { Centrado en operaciones financieras. } \\
\text { Uso de métodos de valoración de mercado (prevalencia de valor } \\
\text { razonable). }\end{array}$ \\
\hline & $r$ & $r$ \\
\hline & $\begin{array}{l}\text { Modelo de gestión relacionado con la } \\
\text { consolidación de economias con rendimientos de } \\
\text { escala creciente. }\end{array}$ & $\begin{array}{l}\text { Modelo de gestión relacionado con la consolidación } \\
\text { y expansión de los mercados financieros. }\end{array}$ \\
\hline
\end{tabular}

Fuente: elaboración propia

\section{Las tensiones presentes entre el ambiente institucional y la realidad organizacional}

El asentamiento de la concepción financiera de la empresa ha sido encabezado en Colombia principalmente por la reconfiguración institucional, antes que por la transformación de la realidad organizacional. Tal 
situación ha ocasionado el surgimiento de tensiones entre los ambientes organizacionales e institucionales, relacionadas con la concepción dominante de empresa.

Desde la década de 1990, el ambiente institucional ha presentado un marcado desplazamiento hacia las tendencias que plantea la financiarización. Estas inclinaciones se han desarrollado de la mano de contingencias como la proliferación de acuerdos de inversión, el aumento de tratados de libre comercio, la flexibilización de los flujos de capital, el respaldo a inversionistas institucionales, la creación de zonas francas, la estandarización de procedimientos aduaneros y la integración regional de la BVC. En conjunto, tales disposiciones han acercado el ambiente institucional colombiano a los discursos dominantes de internacionalización de la empresa, y al concepto de la empresa como un portafolio de activos líquidos. Ahora, la competitividad empresarial se alcanza mediante la gestión financiera eficiente, por lo que un sector financiero próspero es esencial para garantizar la dinámica positiva de la empresa, en particular, y de la economía, en general.

Desde el punto de vista económico, el comportamiento de las actividades agrícolas, manufactureras y financieras ha sido coherente con la transformación del ambiente institucional. En este sentido, durante las últimas décadas la economía nacional ha sido marcada por una reducción de la importancia de las actividades agrícolas y manufactureras, al tiempo que las actividades financieras han obtenido una mayor preponderancia. De hecho, de acuerdo con datos del Banco de la República, entre 1975 y 2015 las actividades manufactureras descendieron del segundo al cuarto renglón en la composición del PIB, al tiempo que las actividades financieras pasaron del tercer al primer renglón del PIB (Pardo, 2016).

En contraste, la aproximación del ambiente organizacional a las dinámicas de la financiarización de la economía se ha visto limitada por la tardanza en la adopción de prácticas empresariales alineadas con la lógica financiera, así como por las características de las pequeñas y medianas empresas (Pymes); ${ }^{8}$ la preponderancia de las actividades primarias y secundarias en el desarrollo económico del país, y la limitada integración del sector empresarial a los mercados financieros.

En primera instancia, la implementación de prácticas de gobierno empresariales vinculadas a una concepción financiera de la firma se empezó a materializar en 2001, en empresas emisoras de valores, ${ }^{9}$ y en 2009 en empresas familiares. Cabe añadir que la implementación de prácticas contables afines a la concepción financiera de la firma se produjo en 2015 y $2016 .^{10}$

En segunda instancia, se destacan cuatro aspectos dentro de los rasgos característicos de las Pymes que han restringido las interrelaciones entre las actividades empresariales y las dinámicas especulativas de los mercados financieros. En ese sentido, se hace referencia a (a) la preferencia por cubrir sus necesidades de financiamiento mediante aportes patrimoniales o acceso a fuentes de liquidez informales; (b) la incapacidad de cumplir los requisitos de solvencia que establece la banca formal; (c) la inexistencia de órganos de gobierno formales, y (c) la ausencia de profesionalización de sus gestores.

En tercera instancia, a pesar de que las actividades agrícolas y manufactureras han decrecido como porcentaje del PIB, la importancia de estas actividades continúa siendo un elemento central para el bienestar económico de la población en general. Así, por ejemplo el sector industrial se mantiene como una de las principales fuentes de empleo formal; ${ }^{11}$ el sector agrícola representa una de las principales opciones de emprendimiento a nivel empresarial, y el sector mineroenergético se ha consolidado desde la primera década del 2000 como impulsor de la economía nacional.

Además, es importante destacar el limitado desarrollo de la Bolsa de Valores de Colombia (BVC), en la cual tan solo cotizaban 69 empresas para el 31 de octubre de 2017. Tal aspecto no solo da cuenta la concentración de la propiedad que caracteriza a las empresas colombianas, sino que refleja simultáneamente la escasa interacción directa que hay entre el ahorro de los hogares y el financiamiento de las actividades organizacionales.

De ese modo, emerge una tensión entre el ambiente institucional y la realidad organizacional, en tanto que el primer aspecto tiende a imponer al segundo una determinada concepción de empresa. De ese modo, se 
insertan a gran escala conceptos y herramientas financieras para la gestión y coordinación de las actividades de trabajo que no necesariamente responden a las necesidades de las organizaciones.

\section{Isomorfismo y gestión del riesgo}

Las perspectivas de inclusión de las empresas colombianas en mercados internacionales se traducen en una oportunidad para alcanzar el ideal de éxito empresarial contemporáneo. Dicho ideal exige ajustes en las dinámicas organizacionales con la finalidad de capitalizar las oportunidades y afrontar las amenazas que plantean mercados con complejidades crecientes. No obstante, los cambios en las prácticas de las organizaciones no obedecen siempre a decisiones estratégicas que buscan fortalecer la eficiencia de la unidad económica en un contexto globalizado; por el contrario, en ocasiones pueden responder además a "un proceso limitador, (conocido como isomorfismo) que obliga a una unidad en una población a parecerse a otras unidades que enfrentan las mismas condiciones ambientales" (Powell y DiMaggio, 1999, p. 107). Una alternativa que implementa para mostrarse como competitiva.

Así, el isomorfismo institucional constituye un componente esencial para explorar las transformaciones de las estructuras formales que procuran la adopción de la concepción financiera de la empresa; una traslación desarrollada con base en las relaciones que emergen de la interacción del ambiente institucional y la realidad organizacional. En este sentido, resulta plausible considerar que la inserción de la concepción financiera de la empresa se apoya en procesos homogenizadores de las prácticas, valores y estructuras organizacionales que reflejan ceremonialmente mitos considerados reflejo de racionalidad en los ambientes institucionales antes que las demandas de las actividades de trabajo (Udy, 1970; Meyer y Rowan, 1999; Gómez, 2009).

De acuerdo con Fennell (1980), y Powell y Dimaggio (1999), el isomorfismo se manifiesta en los niveles de lo competitivo y de lo institucional. Según eso, se centra en la competencia de mercado, el cambio de nichos y las medidas de ajuste implementadas en campos en los que existe competencia libre y abierta. En suma, se ajusta a comportamientos conscientes en los que las organizaciones se transforman para mejorar sus características competitivas. Asimismo, aborda las fuerzas que presionan a las comunidades para adaptarse al mundo exterior; o, en otras palabras, corresponde a comportamientos mediante los que las organizaciones se transforman para adoptar las expectativas de su entorno, y así legitimar sus acciones.

El protagonismo de las finanzas en el desarrollo del modelo económico contemporáneo, junto con la consecuente expansión de las transacciones financieras, han dado lugar precisamente a una mayor complejidad de los ambientes institucionales que originan prácticas y estructuras alineadas con la financiarización económica; factores que, a su vez, han sido apropiados por el sector empresarial, y reproducidos en diversos escenarios. Una de las instituciones racionalizadas en el contexto internacional es la concepción financiera de la empresa, cuyas características son operacionalizadas total o parcialmente al interior de las organizaciones, por cuenta de una aparente necesidad de legitimar la acción empresarial en el ambiente institucional.

De este modo, las tensiones existentes entre la realidad organizacional y el ambiente institucional en Colombia se zanjan con la acción de un isomorfismo institucional. ${ }^{12}$ En el marco de dicho fenómeno, empresas sin intenciones ni potencial para participar en mercados externos, modifican sus formas y prácticas por cuenta de la presión directa e indirecta de un entorno que incentiva procesos estandarizantes como mecanismos de reconocimiento y aprobación.

En este escenario, el dominio y la supervivencia de la financiarización requiere el apoyo de prácticas que provean recursos suficientes para mantener intacto el sentido de inmediatez, el cual define los resultados económicos gestionados por las finanzas. Ante esta situación, desde la década de 1970 se han institucionalizado en el ámbito internacional ${ }^{13}$ diversas prácticas que facilitan (a) la transformación de flujos de ingresos futuros en valores presentes intercambiables; (b) el aumento del flujo de caja hacia los agentes 
financieros; (c) el apalancamiento externo, y (d) el reconocimiento de pérdidas y ganancias no realizadas, entre otros aspectos (Crotty, 2003; Zhang y Andrew, 2014; Laux y Leuz, 2009). Dichas practicas también se manifiestan en el contexto local.

En ese contexto, la proliferación de transacciones económicas fundamentadas en el triunfo del capital financiero ha aumentado la importancia de acciones predictivas y confirmatorias para la toma de decisiones de los poseedores del capital. En consecuencia, han proliferado los escenarios en los que la incertidumbre de las transacciones financieras hace parte integral del devenir organizacional; en efecto, la aplicación de prácticas que permiten identificar, evaluar, tratar y monitorear los riesgos asociados a dicha incertidumbre (Soin y Collier, 2013), emerge como rasgo característico de aquellas organizaciones que se distinguen por su eficiencia en la gestión de un entorno financiarizado.

En el sentido expuesto, la adopción y la implementación del modelo de gobierno corporativo anglosajón, así como de las NIIF, han intensificado en Colombia la transición hacia un marco organizacional que normaliza las características especulativas de los mercados financieros en las dinámicas empresariales; una transformación dada mediante prácticas que sitúan a la administración financiera del riesgo como la característica central de la firma (Soin y Collier, 2013; Zhang y Andrew, 2014). Estos enfoques de contabilidad y de gobierno corporativo "privilegian las dinámicas especulativas que definen a la empresa como una inversión encargada de generar valor en donde [...] resulta imperativo defender la posición de los poseedores del capital sobre las posiciones de los demás agentes" (Pardo, 2015).

En tanto, la modernización de la regulación y de las prácticas contables constituye mecanismos que respaldan la necesidad de modificar algunas conductas de control organizacional, en virtud de políticas y estándares que responden a la incertidumbre derivada de la complejidad financiera observable en las transacciones económicas. De ese modo, la contabilidad adquiere un rol instrumental, pues es utilizada como una herramienta que facilita la apropiación del concepto financiero de la empresa, mediante unas tecnologías de cálculo y una racionalidad particulares; factores que, a su vez, regularizan la incertidumbre y la gestión del riesgo en las organizaciones.

Con el objetivo de ilustrar adecuadamente el papel activo que desempeña la contabilidad en el isomorfismo institucional que privilegia en general la concepción financiera de la empresa, al igual que la gestión del riesgo como principal práctica de control organizacional, en las próximas secciones se abordarán algunos elementos generales de una comprensión particular de la contabilidad. Según lo anterior, esta es entendida como una práctica social e institucional. Asimismo, se describirán algunas de las modernizaciones más importantes que ha traído el ascenso de la contabilidad financiera como subdisciplina dominante.

\section{El papel institucional de las modernizaciones contables}

La implementación de las NIIF en Colombia constituye un camino para la inserción de una concepción financiera de la empresa en la realidad organizacional; una implementación dada por medio del isomorfismo institucional que promueve mediante la estandarización (Gómez Villegas, 2016). De ese modo, elementos externos a la práctica contable han ayudado a institucionalizar una visión hegemónica de la contabilidad, en la que la contabilidad financiera se destaca como la subdisciplina dominante. Simultáneamente, las modernizaciones que tienen lugar en las racionalidades y técnicas contables han contribuido a institucionalizar la conveniencia de las actividades de gestión de riesgo financiero en el ambiente organizacional.

Aquel papel activo, que es atribuido a la contabilidad, se fundamenta en su concepción como una práctica dinámica y socialmente construida (Potter, 2005). Lo anterior significa que es “constitutiva por sí misma, y no de forma derivada o secundaria, de relaciones sociales [...] [de modo que] ejerce una influencia sobre, y es 
influenciada por, una multiplicidad de agentes, agencias, instituciones y procesos" (Miller, 1994, p. 1). ${ }^{14}$ Esta perspectiva de la contabilidad supone el planteamiento de tres elementos característicos.

1. La contabilidad es una tecnología; un medio para cuantificar y hacer visibles los hechos económicos. Consecuentemente, en ella las cualidades se transforman en cantidades, y mediante ella se hacen comparables entidades cuyos procesos y actividades han sido reducidos a una entidad individual. Al definir la forma como los eventos y procesos son visibilizados, las prácticas contables seleccionadas adquieren una capacidad transformadora que actúa sobre las acciones, los individuos y los objetos.

2. La práctica contable está constituida de racionalidades que inciden en la comprensión de la realidad económica que la disciplina busca representar. Estas racionalidades son calculables y comprensibles gracias a la técnica contable; simultáneamente, proveen una base para la difusión de los dispositivos de cálculo de la contabilidad.

3. El dominio económico es construido y reconstruido por cambios en las prácticas de cálculo que promueve la contabilidad. En términos económicos, las prácticas de cálculo hacen comprensibles los procesos, los objetos y las actividades, entre otros aspectos. Sin embargo, dichas prácticas no son estáticas, sino que responden a unas racionalidades que trazan fines y objetivos. Por tanto, cuando estas racionalidades cambian, las prácticas de cálculo también se modifican, y las nociones abstractas mediadas por las representaciones contables son reconstruidas.

Cabe precisar que, al ser la contabilidad una práctica construida socialmente, permite que la adopción de su racionalidad y de algunas de sus técnicas no amerite el respaldo de una regla formal que ratifique la decisión política de un gobierno de acogerla. Al respecto, es preciso señalar que la Ley 1314 de $2009^{15}$ no constituye un punto de inflexión a partir del cual las tecnologías de cálculo, las racionalidades y el dominio económico reproducidos en la contabilidad por la corriente anglosajona ingresan al contexto colombiano. Por el contrario, la existencia de un ambiente macroeconómico afín a la integración de la economía nacional a las transacciones y las tendencias internacionales es un elemento que ha posibilitado, desde la década de 1990 hasta la actualidad, la implementación parcial de las prácticas contables anglosajonas en el contexto colombiano.

Según esta perspectiva, es notorio que existen diferentes vías por las cuales el isomorfismo institucional ha respaldado la aceptación de prácticas contables alineadas con la noción financiera de la empresa y la administración financiera del riesgo. Entre estas vías se destacan (a) la figura de las instituciones normalizadoras internacionales; (b) el papel regulatorio del Estado; (c) el crecimiento de la inversión extranjera en Colombia, y (d) el fortalecimiento de la presencia de las Big Four ${ }^{16}$ (Pardo, 2016).

No obstante, como se ha esbozado previamente, la relación que hay entre el isomorfismo institucional y las modernizaciones de la contabilidad financiera no es de carácter unidireccional. Dicha relación no se reduce a la acción que el isomorfismo ejerce a favor de la aplicación de nociones propias del modelo de contabilidad anglosajón. Además, involucra un papel activo según el cual la contabilidad incentiva el isomorfismo institucional y privilegia la concepción financiera de la empresa, al igual que la gestión del riesgo financiero. Dicha relación será explorada en la siguiente sección del documento.

\section{Las modernizaciones en la contabilidad financiera}

En la economía capitalista, la hegemonía del modelo de acumulación financiero ha propiciado la redefinición de las fronteras de la contabilidad; un contexto en el cual se ha exaltado el rol de la contabilidad financiera como subdisciplina dominante. Esta redefinición se ha caracterizado por la ampliación de los márgenes de la contabilidad mencionada por Miller (1998); así, se permite la inclusión de temas que no eran el objeto de estudio de la disciplina durante el auge del capitalismo industrial. De esta forma, las últimas décadas han 
atestiguado la transformación y la modernización de conceptos, objetivos y técnicas de cálculo consecuentes con un modelo contable estático que pretende maximizar el valor de las inversiones para hacer frente a las expectativas de inversores y acreedores (Archel y Gómez, 2014).

Asimismo, son fácilmente distinguibles en el marco conceptual de la Junta de Normas Internacionales de Contabilidad (IASB, por sus siglas en inglés) algunas de las modernizaciones contables que se alinean con el contexto financiarizado, y que actúan como inductores que legitiman, normalizan e intensifican las transacciones que se desarrollan entre los mercados financieros y las organizaciones. Lo anterior obedece a que en dicho marco se encuentran las definiciones y criterios que integran la racionalidad que determina las tecnologías de cálculo aplicadas al modelo internacional de contabilidad. Dentro de dicho esquema de modernizaciones, se destacan (a) la redefinición de los usuarios de la información financiera, (b) el rol instrumental del valor razonable y (c) la redefinición de las cualidades de la información financiera.

\section{Construcción de los usuarios de la información financiera}

La emisión de normas de contabilidad por parte de organismos anglosajones estandarizantes - especialmente el IASB ${ }^{17}$ - se ha cimentado en procesos reduccionistas que pretenden atender demandas de uniformidad y comparabilidad, en virtud de la necesidad de garantizar información financiera útil para la toma de decisiones en un entorno globalizado. Ante tal escenario, la determinación de los usuarios de la información financiera ha adquirido un rol protagónico en la estructuración y aceptación de los estándares contables, toda vez que contribuye a responder a las demandas de uniformidad y comparabilidad en tanto que se reduce la complejidad de las prácticas contables: objetivo desarrollado en función de las necesidades atribuidas a ciertos grupos de individuos.

De acuerdo con Young (2006), el concepto de usuarios originado en el marco de la información financiera es una construcción abstracta que busca simplificar por medio de un modelo la complejidad que acarrea una amplia variedad de actores. Esta construcción se ha alejado de la categorización amplia que, en la década de $1970,{ }^{18}$ incluía a propietarios, prestamistas, proveedores, potenciales inversionistas y acreedores, empleados, gestores, clientes, analistas financieros, reguladores, sindicatos y al público en general. En contraste, desde el inicio de la década de 2010, ${ }^{19}$ el concepto se ha decantado hacia una categorización estrecha que solo incluye a "los inversores y prestamistas, además de otros acreedores existentes y potenciales" (IASB, 2010, p. 8), es decir poseedores de capital financiero

En ese escenario, la reconfiguración de los usuarios de la información financiera ha reforzado los problemas de asimetría de información que surgen en el contexto organizacional; asimismo, ha impactado la capacidad de rendición de cuentas que históricamente ha distinguido a la disciplina contable. Ahora, la información financiera que provee la contabilidad se estructura con base en conceptos y técnicas en los cuales el agente racional es aquel que se comporta como un poseedor de capital financiero; en los que, además, los agentes en general deben tener formación financiera básica que les permita interpretar adecuadamente la información provista por los estados e informes financieros.

En lo relacionado con la redefinición de la concepción dominante de empresa, esta modernización contable privilegia la concepción financiera, pues subraya los roles de los proveedores del capital financiero. Asimismo, invisibiliza algunos agentes que eran preponderantes en la concepción orgánica de la empresa, y los induce a converger con los intereses y necesidades de inversores, prestamistas y acreedores. De igual forma, fortalece la generación de rentabilidades como vía para alcanzar el excedente económico, toda vez que la toma de decisiones a corto plazo va ligada a la figura del usuario; concepto que cobija a los poseedores del capital financiero. Finalmente, respalda la gestión de riesgo financiero en las organizaciones, lo cual obra en perjuicio de la gestión del riesgo operacional, pues normaliza conceptos como los de la incertidumbre, la tasa de descuento, el valor de la entidad o los fujos de caja futuros. 


\section{El rol instrumental del valor razonable}

La consolidación de los mercados financieros, al igual que de las actividades especulativas como ejes de acumulación de capital, ha reforzado durante las últimas décadas la demanda de métodos de valoración con la capacidad de incluir las dinámicas de los mercados financieros en la realidad organizacional. Así, se hace referencia a una demanda que ha sido atendida por la contabilidad financiera, mediante las propuestas regulatorias de los organismos estandarizadores que cuentan con reconocimiento internacional; entidades que han legitimado y reproducido el desplazamiento de los enfoques de valoración hacia una perspectiva que incorpora expectativas financieras a la representación de la realidad económica de la empresa (Aglietta y Rebérioux, 2009).

El marco normativo del IASB — al igual que el del FASB - ha incentivado la adopción y la aplicación global de métodos de valoración al servicio de los usuarios de la información financiera, de manera tal, que la predicción y la confirmación de transacciones económicas futuras se han convertido en objetivos que trascienden las necesidades de los inversionistas y acreedores, teniendo así un impacto notorio en la organización en su conjunto.

Dentro de estos métodos de valoración se destaca el valor razonable, el cual asigna valores monetarios a los recursos asociados a las actividades empresariales según tres enfoques generales. Dichas perspectivas corresponden al (a) enfoque de mercado, el (b) enfoque del costo y el (c) enfoque del ingreso. Cada uno de ellos busca asegurar que las técnicas aplicadas en el cálculo del valor razonable reflejen las condiciones del mercado, bien sea mediante los precios de las transacciones de mercado, los precios de las transacciones ficticias o las expectativas de mercados sobre las transacciones futuras.

En lo que respecta a los datos utilizados en las técnicas de valoración, la contabilidad financiera da prominencia a los datos de salida y eclipsa los datos de entrada como insumos para la valoración de los componentes de la organización. En lo que atañe a este aspecto, el valor razonable se estructura con base en una jerarquía que, en primera instancia, prioriza el uso de precios en mercados activos; en segunda medida, respalda el uso de precios en mercados no activos e indicadores financieros como tasas de interés y volatilidades implícitas, y, en tercera instancia, sugiere el uso variables no observables que reflejan supuestos de los agentes que realizan la valoración (IASB, 2011). Cabe señalar que, en ocasiones, el cálculo del valor razonable debe ser complementado por ajustes asociados a los riesgos y a la incertidumbre de las variables utilizadas durante la medición.

Así las cosas, la medición al valor razonable refuerza la concepción financiera de la empresa, al privilegiar la selección de datos de salida y la aplicación de técnicas de cálculo financieras. De ese modo, se legitima la noción de que la información más pertinente para representar la realidad organizacional no se sitúa al interior de la organización sino en su entorno.

\section{Redefinición de las cualidades de la información financiera}

En el proceso de actualización que adelanta el IASB sobre la normatividad contable internacional, se destaca el trabajo desarrollado sobre el Marco conceptual para la preparación y presentación de los estados financieros, que fue publicado originalmente en 1989. Dicho trabajo fue renovado en 2010, con lo que se dio origen al Marco conceptual para la información financiera.

El Marco conceptual establece una estructura jerárquica de las características cualitativas que debe tener la información financiera para ser útil en la toma de decisiones Esta estructura jerárquica ubica la relevancia y la representación fiel como las cualidades esenciales, al tiempo que otras cualidades como la comparabilidad, la verificabilidad, la oportunidad y la comprensibilidad son planteadas como características de mejora. De igual 
forma, se nota la desaparición de cualidades como la confiabilidad, la esencia sobre la forma y la prudencia, las cuales hacían parte del Marco conceptual del IASC en 1989.

Con el ánimo de evidenciar la importancia de los cambios de las características cualitativas de la información financiera para la reproducción de la concepción financiera de la empresa, a continuación se analizan algunos elementos de la nueva estructura que plantea el Marco conceptual del IASB.

\section{Relevancia y comprensibilidad}

Según el Marco conceptual del IASB (2010), la relevancia coincide con la facultad que puede tener cierta información para influir en la toma de decisiones de los usuarios; facultad que depende del valor predictivo, del valor confirmatorio y de la materialidad.

Cabe enfatizar que la definición de la información útil, dada en términos de relevancia, así como la de esta, dada a su vez en términos de capacidad predictiva o confirmatoria, no revisten mayor diferencia respecto al marco conceptual de 1989. Sin embargo, adquieren especial importancia cuando son analizadas a la luz de la subordinación emergente de la comprensibilidad ante la relevancia. En este sentido, mientras que el Marco conceptual de 1989 presentaba a la relevancia y la comprensibilidad como cualidades de un mismo nivel, la versión del 2010 evidencia una predilección por la primera sobre la segunda.

El que se sugiera que la información útil debe ser relevante antes que comprensible, brinda mayor libertad para el uso de datos y la aplicación de técnicas que destacan el carácter predictivo y confirmatorio de la información, sin importar la complejidad que reviste. Esta situación aumenta la imperfección y la incompletitud de la información financiera, ${ }^{20}$ bajo una perspectiva organizacional que incluye a todos los stakeholders de la empresa, lo cual se traduce en mayores asimetrías de la información y una reducción del conocimiento común que la contabilidad brinda en beneficio del control en las organizaciones.

En tal escenario, la información clara y concisa que da lugar a la comprensibilidad depende de la evaluación de la relación costo-beneficio que adelanta la organización; un comportamiento organizacional que se facilita y legitima en la medida en que "los informes financieros se preparan para usuarios que tienen un conocimiento razonable de las actividades económicas y del mundo de los negocios" (IASB, 2010, p. A38). De esta forma, el Marco conceptual hace correcta la revelación de información poco comprensible, con base en un discurso que desconoce las diferencias de formación y conocimiento que hay entre los usuarios de la información financiera; concepto de usuarios que, como ya se expuso, ha sido reducida a los inversionistas, prestamistas y acreedores

\section{Representación fiel, prudencia y verificabilidad}

La representación fiel pasó de tener un papel secundario ante la confiabilidad, como constaba en el Marco conceptual de 1989, a tener el carácter de cualidad independiente en el Marco conceptual de 2010; contexto en el cual reemplazó a la confiabilidad. Al respecto, es preciso anotar que este cambio supuso la eliminación de la esencia sobre la forma y la prudencia como características cualitativas de la información financiera. En general, la representación fiel puede ser comprendida como la descripción completa, neutral y libre de error de los fenómenos económicos que la contabilidad busca representar.

Es importante destacar que la explicación completa, neutral y libre de error de los supuestos y métodos aplicados en la representación de un fenómeno económico es suficiente para garantizar fidelidad en la representación de la información financiera. No obstante, tal explicación resulta intrascendente si se pretende evaluar la confiabilidad de la información. En consecuencia, la contabilidad financiera conceptualizada por organismos como el IASB no se interesa por la pertinencia de los métodos aplicados en la descripción de la realidad económica. Por esta razón, es común que las organizaciones recurran, cada vez con mayor frecuencia, 
a información no financiera en las notas de los estados financieros, al igual que en los informes de gobierno corporativo y en los informes de gestión de riesgos, como medio para construir la confianza ausente en la información financiera provista por la contabilidad.

Por otra parte, es importante precisar que la eliminación de la prudencia como característica cualitativa de la información financiera, junto con la subrogación de la verificabilidad al análisis costo-beneficio, legitima en el plano organizacional las valoraciones derivadas de consensos o modelos financieros prospectivos. En ese sentido, la volatilidad y la incertidumbre que distinguen a los mercados y modelos financieros son aceptadas y reproducidas en el escenario organizacional; lo anterior se da mediante la ejecución de prácticas contables caracterizadas por la ausencia de precauciones en los juicios necesarios para realizar estimaciones. Así, se erige una visión de la contabilidad que respalda los datos provistos por los mercados al punto de delegarles la auditoría de los valores de los activos y los pasivos de las organizaciones (Power, 2010).

En resumen, los cambios recientes que han experimentado las características cualitativas del marco conceptual del IASB respaldan la concepción financiera de empresa. En ese sentido, mantiene el foco en las transacciones futuras; facilita la defensa de los modelos financieros, y normaliza la aparición de volatilidades del mercado en los resultados empresariales.

Con el objetivo de ilustrar la construcción teórica que se ha desarrollado en este artículo, la figura 1 expone los vínculos que existen entre la concepción financiera de la empresa y las modernizaciones contables, según ha sido planteado. En tal contexto, estas actúan como la base que permite institucionalizar algunos elementos propios de la financiarización en el plano organizacional, en cuyo caso se hace referencia a aspectos como los poseedores del capital, el valor accionarial y la gestión financiera del riesgo.

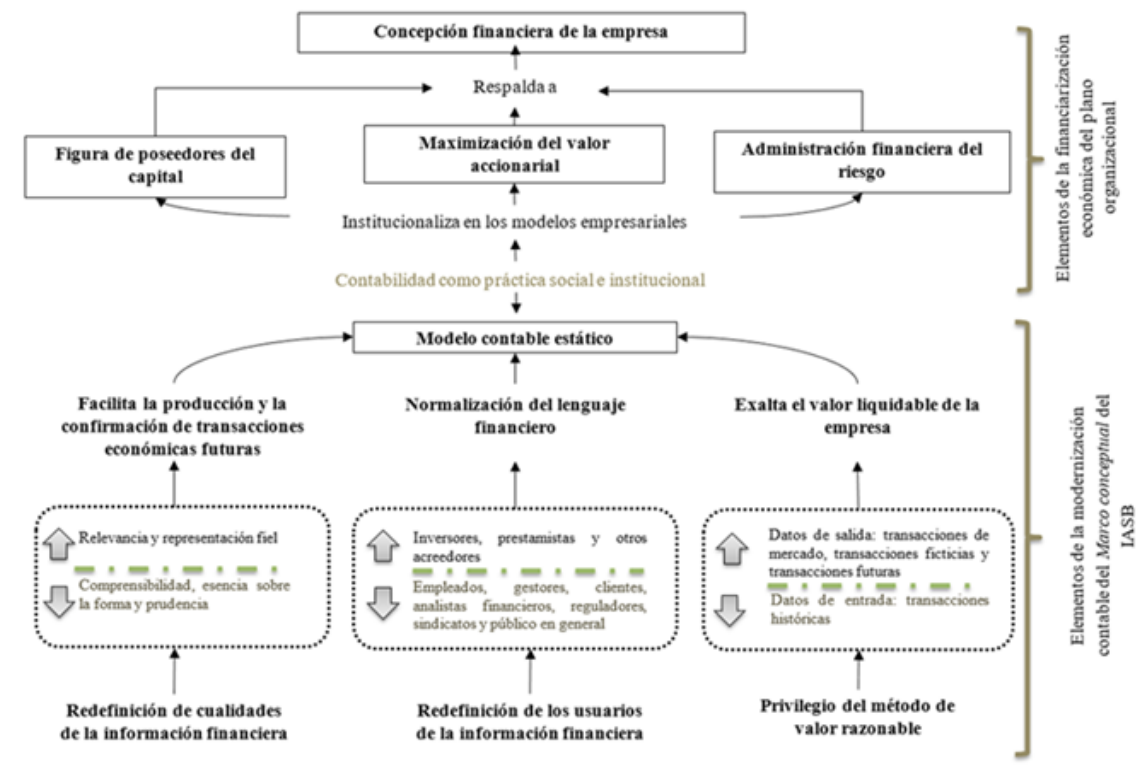

FIGURA 1

Modernizaciones contables a favor de la concepción financiera de empresa Fuente: elaboración propia

\section{Conclusión}

Las reformas macroeconómicas que ha experimentado Colombia durante las últimas décadas, en línea con las medidas impulsadas para Latinoamérica por parte de organismos multilaterales como el Fondo Monetario Internacional y el Banco Mundial, han permitido la estructuración de un ambiente institucional que prioriza la eficiencia financiera en el manejo de los recursos como el elemento que distingue a las 
organizaciones competitivas en un entorno financiarizado. En contraste, el tejido organizacional del país continúa exhibiendo una estructura en la que el desarrollo económico y social se centra en las actividades de los sectores primario y secundario de la economía; consecuentemente, en el contexto nacional, la eficiencia en la gestión de los factores productivos se mantiene como el elemento clave del éxito empresarial.

La situación descrita evidencia la tensión existente entre el ambiente institucional y la realidad organizacional; discordancia que se decanta a favor de la concepción financiera de empresa, como consecuencia de un isomorfismo institucional que lleva a las organizaciones a replicar ceremonialmente determinados mitos de su entorno, en procura de la legitimación de sus actividades. En este escenario, algunas modernizaciones de la contabilidad financiera internacional, apropiadas en el contexto colombiano mediante la adopción parcial o total de normas emitidas por organismos estandarizantes internacionales, han acarreado la implementación de prácticas y racionalidades que realzan los roles de los flujos financieros y la gestión del riesgo como características centrales de las organizaciones modernas.

Modernizaciones contables como la que supone la construcción de los usuarios de la información financiera, el rol instrumental del valor razonable y la redefinición de las características cualitativas de la información financiera, han naturalizado conceptos financieros y comportamientos especulativos en el devenir de las organizaciones. De ese modo, acercan la realidad empresarial colombiana a escenarios en los que la predicción, la confirmación y la incertidumbre constituyen el centro de discusión de las partes interesadas en la compañía; por tanto, se organiza un contexto en el que los informes financieros y no financieros deben dar cuenta de las acciones emprendidas por la empresa para conjurar los riesgos que afrontan los inversionistas, acreedores y prestamistas.

En suma, la exploración realizada denota el papel activo de la contabilidad en la modelación de las prácticas organizacionales a partir de las demandas del ambiente institucional, y plantea la necesidad de cotejar la coherencia entre las prácticas financiarizadas adoptadas por las organizaciones y su aporte a la eficiencia productiva, así como la coherencia de los discursos dominantes sobre gestión del riesgo con las condiciones que deben afrontar las empresas del país.

Como cierre, es preciso enfatizar que las presiones institucionales identificadas en el presente artículo pueden ser abordadas a profundidad mediante revisiones documentales que permitan evaluar el papel performativo que tiene la información financiera y no financiera en la definición de los objetivos, la toma de decisiones y las demandas de control que ejecutan los agentes organizacionales en el contexto colombiano. De igual forma, la exploración de la capacidad endógena de dichas entidades para resistir o adaptarse a las presiones institucionales puede constituir una línea de investigación complementaria; una rama con la capacidad de ampliar el marco de análisis de las relaciones que se entablan entre las instituciones, la contabilidad y las organizaciones.

\section{Referencias}

Aglietta, M. y Rebérioux, A. (2009). El debate contable. En F. Arbeláez (trad.), El capitalismo financiero a la deriva (pp. 151-182). Bogotá: Universidad Externado.

Archel, P. y Gómez, M. (2014). Crisis de la valoración contable en el capitalismo cognitivo. Innovar, 24(52), 103-116.

Banco Interamericano de Desarrollo y Bolsa de Valores de Colombia. (2009). Desarrollo de instrumentos informativos sobre mercados de capitales. Bogotá: Colombia Capital.

Boyer, R. (2007). Assessing the impact of fair value upon financial crises. Socio Economy Review, 5(4) 779-807.

Cañibano, L. (1988). Contabilidad. Análisis contable de la realidad económica. Madrid: Ediciones Pirámide.

Crotty, J. (2003). The neoliberal paradox: The impact of destructive product market competition and 'modern'financial markets on nonfinancial corporation performance in the neoliberal era. Review of Radical Political Economics, 35(3), 271-279. 
Departamento Nacional de Planeación. (2005). Estadísticas históricas de Colombia. Recuperado de https://www.dnp. gov.co/estudios-y-publicaciones/estudios-economicos/Paginas/estadisticas-historicas-de-colombia.aspx

Fennell, M. (1980). The effects of environmental characteristics on the structure of hospital clusters. Administrative Science Quarterly, 25(3), 485-510.

Financial Accounting Standard Board. (1978). Financial Accounting Concepts (N.o 1). Recuperado de http://fasb.o rg/jsp/FASB/Page/PreCodSectionPage\&cid=1176156317989

Franco, M. y Urbano, D. (2010). El éxito de las Pymes en Colombia: un estudio de casos en el sector salud. Estudios Gerenciales, 26(114), 77-97.

Frieden, J. (2006). Capitalismo global. El trasfondo económico de la historia del siglo XX. Barcelona: Crítica.

Gaitán, S. (2010). Gobierno corporativo en Colombia: tendencias actuales. Administer, (15), 137-153.

García, A. y Gómez, J. (2009). Determinantes de las fusiones y adquisiciones en el sistema financiero colombiano. 1990-2007. Borradores de Economia, (550), 1-29.

Giraldo, C. (2007). ¿Protección o desprotección social? Bogotá: Desde Abajo.

Gómez, M. (2009). Los informes contables externos y la legitimidad organizacional con el entorno: estudio de un caso en Colombia. Innovar, 19(34), 147-166.

Gómez Villegas, M. (2016). NIIF y PYMES retos de la contabilidad para el contexto y la productividad. Cuadernos de Administracióm, 29(53), 49-76. https://doi.org/10.11144/Javeriana.cao29-53.nmrc

Guttman, R. (2009). Una introducción al capitalismo introducido por las finanzas. Ola financiera, 2(2) 20-59.

Harvey, D. (2006). Spaces of global capitalism. United Kingdom: Verso.

International Accounting Standrad Board. (2010). Marco conceptual para la información financiera. IFRS Foundation. Recuperado de https://www.mef.gob.pe/contenidos/conta_publ/con_nor_co/no_oficializ/ES_GVT_RedBV 2016_conceptual.pdf

International Accounting Standrad Board, Norma Internacional de Información Financiera 13a de 2011, Medición del valor razonable $₫$ Londres, International Financial Reporting Standards Foundation. Recuperado de https://ww w.mef.gob.pe/contenidos/conta_publ/con_nor_co/no_oficializ/nor_internac/ES_GVT_IFRS13_2013.pdf

Laux, C. y Leuz, C. (2009). The crisis of fair-value accounting: Making sense of the recent debate. Accounting, Orranizations and Society, 34, 826-834.

Ley 964 de 2005, Congreso de Colombia $₫$ Diario Oficial $45 \# 63$ del 8 de julio de 2005, Bogotá.

Ley 1314 de 2009, Congreso de Colombia $§$ Diario Oficial $47 \# 109$ de julio 13 de 2009, Bogotá.

López, L. F. (2010). Transformación productiva de la industria en Colombia y sus regiones despúes de la apertura. Cuadernos de Economía, 29(53), 239-284.

López, A. (1995). Las últimas décadas: un proceso lento e interrumpido de liberación financiera. Borradores semanales de economia, (27), 1-47.

Lordon, F. (2007). Los fondos depensiones, ¿una trampa para zoquetes? El espejismo de la democracia accionarial. Bogotá: Universidad Externado de Colombia.

Meyer, J. y Rowan, B. (1999). Organizaciones institucionalizadas: la estructura formal como mito y ceremonia. En W. Powel y P. Dimaggio (comps.), El nuevo institucionalismo en el análisis organizacional (79-103). México: Fondo de Cultura Económica-Colegio Nacional de Ciencias Políticas y Administración.

Miller, P. (1994). Accounting as social and institutional practice: An introduction. En A. Hopwood y P. Miller (eds.), Accounting as social and institutional practice (pp. 1-19). New York: Cambridge University.

Miller, P. (1998). The margins of accounting. European Accounting Review, 46(1) 605-621.

Misas Arango, G. (1998). Colombia: la estrategia empresarial en la apertura. En W. Peres (ed.), Grandes empresas y grupos industriales latinoamericanos. Expansión y desafios en la era de la apertura y la globalización (pp. 333-396). México, D.F.: Cepal-Siglo XXI Editores. 
Pardo, S. (2014). Una evaluación de la relación entre la disciplina contable y la evolución del sistemapensional colombiano, a partir de la ley 100 de 1993 (Tesis de maestría en Administración, Universidad Nacional de Colombia, Bogotá, Colombia). Recuperado de http://bdigital.unal.edu.co/46657/1/08941016.2014.pdf

Pardo, S. (2015). Prácticas de valoración y gobierno corporativo: medios para la resignificación del control en las organizaciones colombianas. Cuadernos de Contabilidad, 16(40), 205-231.

Pardo, S. (2016). Prácticas contables y de gobierno corporativo: facilitadoras del ascenso de la concepción financiera de empresa en Colombia. Contaduria Universidad de Antioquia, (69), 155-172.

Potter, B. (2005). Accounting as a social and institutional practice: perspectives to enrich our understanding of accounting change. Abacus, 41(3), 265-289.

Powell, W. y DiMaggio, P. (1999). Retorno a la jaula de hierro: el isomorfismo institucional y la racionalidad colectiva en los campos organizacionales. En W. Powell (ed.), El nuevo institucionalismo en el análisis organizacional (pp. 104-125). México: Fondo de Cultura Económica.

Power, M. (2010). Fair value accounting, financial economics and the transformation of reliability. Accounting and Business Research, 40(6), 197-210.

Prebisch, R. (1967). Hacia una dinámica del desarrollo latinoamericano. Montevideo: Ediciones de la Banda Oriental.

Rayman, R. (2007). Fair value accounting and the present value fallacy: The need for an alternative conceptual framework. The British Accounting Review, 39(3), 211-225.

Resolución 275 de 2001, Ministerio de Hacienda y Crédito Público $₫$ Ministerio de Hacienda y Crédito Público, Bogotá. Recuperado de http://www.notinet.com.co/pedidos/res-275-01.doc

Saavedra, M. y Bustamante, S. (2013). El problema de financiamiento de la PYME y el sistema nacional de garantías en Colombia. Contaduria Universidad de Antioquia, (62), 69-88.

Soin, K. y Collier, P. (2013). Risk and risk management in management accounting and control. Management Accounting Research, 24(2), 82-87.

Sunder, S. (2002). Knowing what others know: Common knowledge, accounting, and capital markets. Accounting Horizons, 16(4), 305-318.

Udy, S. H. (1970). Work in traditional and modern society. Englewood Cliffs, NJ: Prentice Hall.

Young, J. (2006). Make up users. Accounting, Organizations and Society, 31(6), 579-600.

Zhang, Y. y Andrew, J. (2014). Financialisation and the conceptual framework. Critical Perspectives on Accounting, 25(1), 17-26.

\section{Notas}

1 Concepto que será desarrollado en la segunda sección del documento.

2 En Latinoamérica, El modelo ISI fue influenciado por las posturas de política económica de la Comisión Económica para América Latina y el Caribe (CEPAL). Según ello, se destacan el pensamiento de Raúl Prebisch (1967) y su teoría de la dependencia.

3 Los trabajadores gozaban de un salario medio bajo y sus capacidades se concentraban todavía en actividades manufactureras.

4 Dentro de este conjunto de reformas se destacan la Ley 45 de 1990, que dictó disposiciones para regular el sector financiero y asegurador; la Ley 50 de 1990, que introdujo reformas al código sustantivo del trabajo; la Ley 9 de 1991, que disminuyó los trámites burocráticos y eliminó el monopolio sobre la tenencia de divisas por parte del Banco de la República (López, 1995); y la Ley 100 de 1993, que reformó el sistema de seguridad social mediante la introducción de entidades privadas encargadas de la administración de los aportes a los sistemas de salud y pensión.

5 Durante la década de 1990, el Estado puo en venta las participaciones que tenía en instituciones financieras como el Banco Ganadero, el Banco Tequendama o el Banco Popular. De ese modo, incentivó el ingreso de instituciones financieras internacionales al país como el BBVA y el Banco Santander, y promovió el crecimiento de conglomerados financieros como el grupo Aval.

6 Este argumento desconoce la diferencia existente entre el accionista-inversor y el accionista-propietario, cada uno de los cuales posee características e intereses particulares. El accionista-inversor se interesa por la especulación y los rendimientos 
de largo plazo. En tanto, el accionista-propietario se preocupa por la operación real de la empresa, puesto que su interés se centra en los rendimientos de largo plazo.

7 La aplicación del valor razonable, según el paradigma de la contabilidad, y en contraste del valor presente neto, de acuerdo con el de las finanzas, es ejemplo de las prácticas que imprimen volatilidad a la valoración de la firma. En consecuencia, dichas prácticas incentivan prácticas especulativas en los accionistas-inversores.

8 Las Pymes componen el 96\% de las empresas del país (Franco y Urbano, 2010; Saavedra y Bustamante, 2013). Dicho porcentaje no difiere sustancialmente ante los que se observan en otros países.

9 Lo descrito comenzó a suceder tras la emisión de la Resolución 275 de 2001 y de la Ley 964 de 2005 (BID y BVC, 2009; Gaitán, 2010).

10 Periodo en el que las empresas se vieron obligadas a presentar estados financieros de apertura bajo la jurisdicción de las NIIF.

11 Según datos publicados por el Departamento Nacional de Planeación en el año 2005, el sector industrial proveía alrededor de $1 \$ 00 \# 00$ empleos en las 13 ciudades más importantes del país; tal cifra solo es superada por las que registran los sectores de comercio y servicios comunales (DNP, 2005).

12 De acuerdo con Dimaggio y Pawell (1999), existen tres mecanismos no excluyentes por medio de los cuales una empresa reproduce las características y estructuras de otras organizaciones, con la finalidad de alcanzar legitimidad en su entorno. Dichos mecanismos son (a) el isomorfismo coercitivo, cuyos fundamentos son las relaciones de dependencia entre organizaciones; (b) el isomorfismo mimético, que se basa en la incertidumbre y la imitación de organizaciones líderes; y (c) el isomorfismo normativo, el cual se relaciona con la profesionalización y el papel de los agentes especializados en la prescripción de acciones.

13 Inicialmente en los países anglosajones, pero posteriormente en todas las regiones del mundo.

14 "Accounting has come to be regarded a social and institutional practice, one that is intrinsic to, and constitutive of social relations, rather than derivative or secondary [...] in which accounting exerts an influence on, and in turn is influenced by, a multiplicity of agents, agencies, institutions and processes" (Miller, 1994, p. 1)

15 Mediante dicha legislación se regulan los principios y las normas de contabilidad de información financiera, así como del aseguramiento de la información, que son aceptados en Colombia. Asimismo, se señalan las autoridades competentes para su expedición, al igual que el procedimiento idóneo que las cobija, y se determina qué entidades están encargadas de vigilar su cumplimiento.

16 Esta expresión hace referencia a las cuatro corporaciones de auditoría más grandes del mundo: PricewaterhouseCoopers (PWC), Deloitte \& Touche, KPMG y Ernst \& Young (EY).

17 Se destaca también el papel del FASB y el soporte de los organismos estandarizantes en los procesos de aseguramiento de la información financiera.

18 Lo descrito puede constatarse en el Statement of Financial Accounting Concepts N.o 1 del Financial Accounting Standard Board (FASB) que fue emitido en 1978.

19 Lo descrito puede constatarse en la versión del 2010 del Marco Conceptual del International Accounting Standrad Board (IASB).

20 De acuerdo con (Sunder, 2002), la información imperfecta surge cuando las reglas o las estructuras son de conocimiento común entre los participantes de un sistema, pero no se conocen las acciones y eventos de otros participantes. Por su parte, la información incompleta surge cuando las reglas y las estructuras no son de conocimiento común entre los participantes del sistema.

* Artículo de revisión.

\section{Licencia Creative Commons CC BY 4.0}

Para citar este articulo: Pardo-López, S. y Peña Cortés, Á. R. (2017). La contabilidad financiera como motor de la redefinición de la concepción de empresa. Cuadernos de Contabilidad, 18(46). 1-21. https:// doi.org/10.11 144/Javeriana.cc18-46.cfmr 